UG-3/99

SPIN-99/03

February, 1999

\title{
Kaluza-Klein Monopole and 5-brane Effective Actions
}

\author{
Eduardo Eyras f \\ Institute for Theoretical Physics \\ University of Groningen \\ Nijenborgh 4, 9747 AG Groningen, The Netherlands \\ and \\ Yolanda Lozano 3 \\ Spinoza Institute \\ University of Utrecht \\ Leuvenlaan 4, 3508 TD Utrecht, The Netherlands
}

\begin{abstract}
We review the construction of the Kaluza-Klein monopole of the Type IIA theory in the most general case of a massive background, as well as its relation via T-duality with the Type IIB NS-5-brane. This last effective action is shown to be related by S-duality to the D5-brane effective action.
\end{abstract}

\footnotetext{
${ }^{1}$ To appear in the proceedings of the 32nd Symposium Ahrenshoop on the Theory of Elementary Particles, Buckow, Germany, September 1998.

${ }^{2}$ E-mail address: E.A.Eyras@phys .rug.nl

${ }^{3}$ E-mail address: Y.Lozano@phys .uu.nl
} 


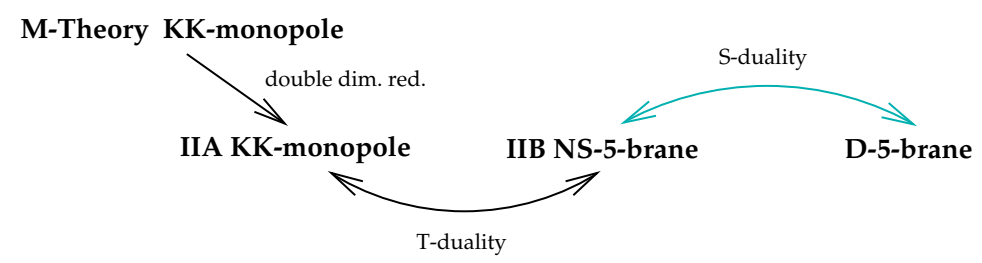

Figure 1: Derivation of the different actions considered in this paper. The (massive) IIA KK-monopole is obtained from the (massive) M-theory KK-monopole after a double dimensional reduction. Applying now $T$-duality in the IIA KK-monopole action the Type IIB NS-5-brane action is obtained, and it is $S$-dual to the D5-brane effective action.

The most general Type IIA superstring theory contains a mass parameter dual to the RR 9-form potential to which the D8-brane couples minimally [1], the corresponding low energy effective action being the massive IIA supergravity of Romans [2]. An eleven dimensional interpretation of this theory can only be found when the eleventh direction is compact [3], with Lorentz invariance taking place in the other ten dimensional coordinatesf. The explicit eleven dimensional supergravity action depends on the Killing vector associated to translations along the eleventh coordinate, and gives the massive Type IIA supergravity action of Romans after a direct dimensional reduction along this direction. The effective actions of the M-branes of this theory are described by gauged sigma-models in which the Killing isometry is gauged, with a gauge coupling constant proportional to $m$. Two massive branes in the Type IIA theory can be obtained from any such M-brane depending on whether we perform a direct or a double dimensional reduction. In particular the massive D6-brane is obtained by dimensional reduction along the Taub-NUT direction of a massive M-KK-monopole in which the two Killing vectors associated to the mass and the Taub-NUT isometries coincide [5]. The construction of a massive KK-monopole in eleven dimensions is more subtle than that of an ordinary brane, since the monopole is already described by a gauged sigma-model in the massless case. The massless Mtheory KK-monopole [6] behaves like a 6-brane, and its field content is that of the 7 -dimensional vector multiplet, involving 3 scalars and 1 vector. Since the embedding coordinates describe 11-7=4 degrees of freedom one scalar has to be eliminated by gauging an isometry of the background. This isometry is that associated to translations of the Taub-NUT direction of the transverse space to the monopole. The massive M-KK-monopole of [5 cannot give rise to a massive Type IIA KK-monopole after double dimensional reduction, since the gauged isometry disappears in the reduction. One needs

\footnotetext{
${ }^{4}$ This circumvents the no-go theorem of $[$ 国.
} 
to construct a massive M-KK-monopole in which the two isometry directions associated to the mass and the Taub-NUT space are different. Double dimensionally reducing along the direction associated to the mass will give rise to the effective action of the massive IIA KK-monopole. This explicit effective action has been constructed in [7]. Here we just summarize its worldvolume field content and the interpretation of these fields in terms of solitons in the brane.

\begin{tabular}{|c||l|l|l|l|l|l|}
\hline M-theory KK & & $\hat{\omega}_{\hat{\imath}}^{(1)}$ & $\hat{d}_{\hat{\imath}_{1} \ldots \hat{\imath}_{5}}^{(5)}$ & & $\hat{\omega}_{\hat{\imath}_{1} \ldots \hat{\imath}_{6}}^{(6)}$ & $\hat{\omega}_{\hat{\imath}_{1} \ldots \hat{\imath}_{7}}^{(7)}$ \\
\hline IIA KK & $\omega^{(0)}$ & $\omega_{i}^{(1)}$ & $d_{i_{1} \ldots i_{5}}^{(5)}$ & $\omega_{i_{1} \ldots i_{5}}^{(5)}$ & $\omega_{i_{1} \ldots i_{6}}^{(6)}$ & \\
\hline
\end{tabular}

Table 1: Worldvolume field content of the massive M-theory and Type IIA KK-monopoles. In this table we summarize the worldvolume fields present in the effective actions of the massive M-theory and massive IIA KK-monopoles.

We find, as in the massless case, a 1-form $\hat{\omega}^{(1)}$ describing a 0 -brane soliton in the worldvolume of the KK-monopole. Its dual 4-form describes a 3-brane soliton. They correspond to the intersections: $(0 \mid \mathrm{M} 2, \mathrm{MKK})$ and (3|M5, MKK), respectively. The monopole contains as well a 4-brane soliton which couples to the 5 -form dual to one of the embedding scalars:

$(4 \mid \mathrm{MKK}, \mathrm{MKK})_{1,2}$. All these intersections have been already discussed in the literature for the massless monopole [8].

In the massive case we find a new field $\hat{d}^{(5)}$ which couples to the 5-brane soliton represented by the configuration:

$$
(5 \mid \mathrm{M} 9, \mathrm{MKK})=\left\{\begin{array}{c|ccccccccccc}
\times & \times & \times & \times & \times & z & - & \times & \times & \times & \times \\
\times & \times & \times & \times & \times & \times & \times & z & - & - & -
\end{array}\right.
$$

The 5-brane soliton predicted by the M-KK and M9-brane worldvolume supersymmetry algebras is realized as a 4-brane soliton given that it cannot develop a worldvolume direction along the isometry of the M9-brane. This is in agreement with the worldvolume field content that we have found for the massive M-KK-monopole, since the only worldvolume field to which this soliton can couple is the 5 -form $\hat{d}^{(5)}$.

The 6 -form $\hat{\omega}^{(6)}$ is interpreted as the tension of the monopole and couples to the 5-brane soliton realized as the embedding of an M5-brane on the

\footnotetext{
${ }^{5}$ We use here a notation where $\times(-)$ indicates a worldvolume (transverse) direction. The first $\times$ in a row indicates the time direction. The $z$-direction in the monopole corresponds to the isometry direction of the Taub-NUT space. A single M9-brane contains as well a Killing isometry in its worldvolume, as has been discussed in [9, 10]. This isometric direction has been depicted as well as a $z$-direction.
} 
KK-monopole. Finally, $\hat{\omega}^{(7)}$ couples to the 6-brane soliton describing the embedding of the monopole in an M9-brane: (6|M9, MKK). From the point of view of the M9-brane this 6-brane soliton couples to the dual of its 1-form vector field [10].

The double dimensional reduction of the action of the massive M-theory KK-monopole gives that of the massive IIA KK-monopole, and it is constructed explicitly in [7]. Its worldvolume field content is summarized in Table 1.

The worldvolume fields that couple to the soliton solutions of a KKmonopole are those necessary to construct invariant field strengths for the fields $i_{k} C^{(p+1)}$ (see [11]). These field strengths have the form:

$$
\mathcal{K}^{(p)}=p \partial \omega^{(p-1)}+\frac{1}{2 \pi \alpha^{\prime}}\left(i_{k} C^{(p+1)}\right)+\ldots,
$$

so that $\omega^{(p-1)}$ couples to a $(p-2)$-brane soliton which describes the boundary of a $p$-brane ending on the monopole, with one of its worldvolume directions wrapped around the Taub-NUT direction of the monopole. The target space field associated to $\omega^{(1)}$ is $\left(i_{k} C^{(3)}\right)$ so that it describes a wrapped D2-brane ending on the monopole. It is worth noting that the Born-Infeld field does not couple to the action, which reflects the fact that there are no fundamental strings ending on the monopole.

As in the massless case, we find the configurations: $(2 \mid \mathrm{D} 4, \mathrm{KK}),(3 \mid \mathrm{NS} 5, \mathrm{KK})$ and $(3 \mid \mathrm{KK}, \mathrm{KK})_{1,2}[8]$. When the mass is non-zero there is a new worldvolume field $d^{(5)}$ (associated to dual massive transformations), which couples to a 4-brane soliton. This soliton is a domain wall in the six dimensional worldvolume and is described by the configuration:

$$
(4 \mid \mathrm{D} 8, \mathrm{KK})=\left\{\begin{array}{c|ccccccccc}
\times & \times & \times & \times & \times & - & \times & \times & \times & \times \\
\times & \times & \times & \times & \times & \times & z & - & - & -
\end{array}\right.
$$

which is obtained by reducing the $(4 \mid \mathrm{M} 9, \mathrm{KK})$ soliton configuration of the M-theory KK-monopole along the isometric direction of the M9-brane. This intersection is related by T-duality to various intersections considered in the literature. There is as well another 4-brane soliton in the KK-monopole worldvolume which is already present in the massless case. This is the embedding of the D4-brane on the monopole: (4|D4, KK), and it couples to the worldvolume field $\omega^{(5)}$, describing the tension of the monopole. Finally, the reduction of the 6-brane soliton $(6 \mid \mathrm{M} 9, \mathrm{KK})$ gives a 5 -brane soliton coupled to $\omega^{(6)}$ which is realized as the embedding of the KK-monopole on a KK-7A-brane国.

\footnotetext{
${ }^{6}$ This brane is obtained by reducing the M9-brane along a worldvolume direction other
} 
We can now construct the effective action of the Type IIB NS-5-brane by performing a (massive) T-duality transformation in the IIA KK-monopole along its Taub-NUT direction. The details of this calculation are presented in [11]. The result is the following effective action:

$$
\begin{aligned}
S= & -T_{\mathrm{B} 5} \int d^{6} \xi e^{-2 \varphi} \sqrt{1+e^{2 \varphi}\left(C^{(0)}\right)^{2}} \sqrt{\left|\operatorname{det}\left(g-\left(2 \pi \alpha^{\prime}\right) \frac{e^{\varphi}}{\sqrt{1+e^{2 \varphi}\left(C^{(0)}\right)^{2}}} \tilde{\mathcal{F}}\right)\right|} \\
& +\frac{1}{6 !}\left(2 \pi \alpha^{\prime}\right) T_{\mathrm{B} 5} \int d^{6} \xi \epsilon^{i_{1} \ldots i_{6}} \tilde{\mathcal{G}}_{i_{1} \ldots i_{6}}^{(6)} .
\end{aligned}
$$

$\tilde{\mathcal{F}}$ is defined as $\tilde{\mathcal{F}}=2 \partial c^{(1)}+1 /\left(2 \pi \alpha^{\prime}\right) C^{(2)}$ and $\tilde{\mathcal{G}}^{(6)}$ is the gauge invariant Wess-Zumino term:

$$
\begin{aligned}
\tilde{\mathcal{G}}^{(6)}= & \left\{6 \partial \tilde{c}^{(5)}-\frac{1}{2 \pi \alpha^{\prime}} \tilde{\mathcal{B}}-\frac{45}{2\left(2 \pi \alpha^{\prime}\right)} \mathcal{B} C^{(2)} C^{(2)}-15 C^{(4)} \tilde{\mathcal{F}}\right. \\
& -180\left(2 \pi \alpha^{\prime}\right) \mathcal{B} \partial c^{(1)} \partial c^{(1)}-90 \mathcal{B} C^{(2)} \partial c^{(1)} \\
& \left.+15\left(2 \pi \alpha^{\prime}\right)^{2} \frac{C^{(0)}}{e^{-2 \varphi}+\left(C^{(0)}\right)^{2}} \tilde{\mathcal{F}} \tilde{\mathcal{F}} \tilde{\mathcal{F}}\right\} .
\end{aligned}
$$

This action is S-dual to the D5-brane effective action, with the worldvolume fields transforming as $\mathrm{SL}(2, \mathrm{Z})$ doublets. It is interesting to note that the BI field transforms into a 1 -form $c^{(1)}$, and not into a 3 -form as one would have expected. This could be useful for the construction of (p,q) 5-branes.

Analogously to this construction it is possible to derive the effective action of the Type IIB KK-monopole by performing a T-duality transformation in the effective action of the IIA NS-5-brane (see [11]). These results show explicitly the duality relations between the various 5-branes of the IIA and IIB theories.

\section{Acknowledgements}

Y.L. would like to thank the organizers of the 32nd Symposium Ahrenshoop on the Theory of Elementary Particles, Buckow (Germany), for giving her the opportunity to present this material. The work of E.E. is part of the research program of the Dutch Foundation FOM.

\section{References}

than the $z$-direction, but it is not predicted by the Type IIA supersymmetry algebra [12]. As discussed in [10], this is also the case for the KK-6A brane, obtained by reducing the M-KK-monopole along a transverse direction different from the Taub-NUT direction. These branes are required by U-duality of M-theory on a d-torus (see for instance [13]). 
[1] E. Bergshoeff, M. de Roo, M. B. Green, G. Papadopoulos and P. K. Townsend, Nucl. Phys. B470 (1996) 113, hep-th/9601150;

J. Polchinski, Phys. Rev. Lett. 75 (1995) 4724, hep-th/9510017.

[2] L.J. Romans, Phys. Lett. B169 (1986) 374.

[3] E. Bergshoeff, Y. Lozano and T. Ortín, Nucl. Phys. B518 (1998) 363, hep-th/9712115.

[4] K. Bautier, S. Deser, M. Henneaux and D. Seminara, Phys. Lett. B406 (1997) 49, hep-th/9704131.

[5] E. Bergshoeff, E. Eyras and Y. Lozano, Phys. Lett. B430 (1998) 77, hep-th/9802199.

[6] E. Bergshoeff, B. Janssen and T. Ortín, Phys. Lett. B410 (1997) 132, hep-th/9706117.

[7] E. Eyras and Y. Lozano, hep-th/9812188, to appear in Nucl. Phys. B.

[8] E. Bergshoeff, M. de Roo, E. Eyras, B. Janssen and J.P. van der Schaar, Class. Quant. Grav. 14 (1997) 2757, hep-th/9704120;

E. Bergshoeff, J. Gomis and P.K. Townsend, Phys. Lett. B421 (1998) 109, hep-th/9711043;

G. Papadopoulos, Phys. Lett. B434 (1998) 277, hep-th/9712162.

[9] E. Bergshoeff and J.P. van der Schaar, hep-th/9806069.

[10] E. Eyras and Y. Lozano, Contribution to the proceedings of the TMRmeeting Quantum Aspects of Gauge Theories, Supersymmetry and Unification, Corfu, September 1998, hep-th/9812225.

[11] E. Eyras, B. Janssen and Y. Lozano, Nucl. Phys. B531 (1998) 275, hep-th/9806169.

[12] C.M. Hull, Nucl. Phys. B509 (1998) 216, hep-th/9705162.

[13] N. Obers and B. Pioline, hep-th/9809039. 